\title{
Lipschitz Stability for Hyperbolic Inequalities in Octants With the Lateral Cauchy Data and Refocising in Time Reversal
}

\author{
Michael V. Klibanov \\ Department of Mathematics and Statistics, \\ University of North Carolina at Charlotte, \\ Charlotte, NC 28223, U.S.A. \\ E-mailmklibanv@email.uncc.edu
}

September 29, 2005

\begin{abstract}
Hyperbolic equations and inequalities in octants with the lateral Cauchy data at coordinate palnes are consdered.Lipschitz stability estimate is established in the case when both the inhomogeneous right hand side and (unknown) initial conditions at $\{t=0\}$ have a finite support. This is the first stability estimate for such a Cauchy problem in an infinite domain. Refocusing of time reversal fields in octants follows. It is shown that the modified Quasi-Reversibility Method can be applied for the numerical solution of such a Cauchy probem including computational time reversal.
\end{abstract}

\section{Introduction}

Let $n=2$ or 3 and $O_{n}=\left\{x_{1}, \ldots, x_{n}>0\right\} \subset R^{n}$ be the positive octant. A heterogeneous hyperbolic equation and, more generally a hyperbolic inequality in $O_{n}$ is considered. Instead of initial conditions at $\{t=0\}$ which are assumed to be unknown, lateral Cauchy data are given at finite parts of coordinate hyperplanes $\left\{x_{i}=0\right\} \cap \partial O_{n}$. It is assumed that both the inhomogeneous right hand side and unknown initial conditions have a compact support in $O_{n}$. The main result of this short communication is the Lipschitz stability estimate of this Cauchy problem. The author is not aware about previous similar results in unbounded domains. In [10], [11] and [14] such theorems were established for bounded domains: the case of a hyperbolic equation was considered in [10] and [14] and the case of a hyperbolic inequality was considered in [11]. Proofs are basically the same for both cases and are based on the Carleman estimate for the operator $\partial_{t}^{2}-c^{2} \Delta$; further developments and more references can be found in [9] and [16]; the case of the nonconservative Schrödinger equation 
was considered in [17] and [18] (also, see references cited there). Such a Lipschitz stability estimate is often called the "exact observability theorem" in the control theory [17], [18].

This estimate implies both the Lipschitz stability and the uniqueness of the inverse problem of determining the unknown initial condition at $\{t=0\}$. Solution of this inverse problem, in turn provides solution of the problem of computational time reversal (see the end of section 2). The author believes that the problem of the computational time reversal of a wave field is a particular case of the Cauchy with the lateral data for a hyperbolic equation and, consequently, of the correspondong inverse problem of determining initial conditions in such an equation. It was Dr. Alexandre Timonov who first draw the author's attention to this connection. Therefore, the Lipschitz stability estmate of this paper implies that the time reversed wave field in an octant refocuses near orignal point-like sources, i.e., the solution of that Cauchy problem replicates the $\delta$-like sources near $\{t=0\}$. To investigate refocusing , we introduce a formal definition first (section 5). It is shown that refocusing of the time reversed wave field follows from the Lipschitz stability for a corresponding Cauchy problem for a hyperbolic equation with the lateral Cauchy data. A different idea of the proof of refocusing is presented in [2] and [3]. Experimentally refocusing was observed in, e.g., [6] (also, see references cited there). We refer to [1], [4], [5] and references cited there for studies of the computational time reversal in random media. Also, see [1], [7], [8] and references cited there for a variety of approaches to computational time reversal. Note that previous works on computational time reversal did not include the attenuation term and the heterogeneous right hand side of a hyperbolic equation, and coefficients of governing equations were not assumed to be dependent on both spatial and time variables.

The Lipschitz stability estimate enables one to apply a 'hyperbolic' analog of the QuasiReversibility Method (QRM) [19] for a stable numerical solution of this inverse problem, i.e., for the computational time reversal. This version of the QRM is a special case of the Tikhonov regularizing functional [20]. Convergence of this analog of the QRM was proven in [10], [12] and [14] and numerical experiments were conducted in [12]. These experiments have demonstrated a good stability of this method, at least for those examples which were considered in [12]. It was proposed in [13] and [14] to apply the hyperbolic version of the QRM to the computational time reversal in a bounded domain. As to the computational time reversal in the half space, it was proposed in [15] to solve this problem via the solution of the inverse problem of the determination of initial conditions (in a context which is slightly different from one of this article) using the Laplace transform and the convexification method. The concept of a close connection between the problem of time reversal and the inverse problem of the determination of initial conditions in a hyperbolic equation was first presented to the author by Dr. Alexandre Timonov; also see [2] and [3] for a similar concept.

\section{Statements of Problems}

Let $R, T=$ const. $>0$. Denote $D_{T}^{n+1}=R^{n} \times(0, T), n=2,3$. Below the function 
$u(x, t) \in H^{2}\left(D_{T}^{n+1}\right)$. First, we consider the following hyperbolic Cauchy problem

$$
\begin{gathered}
u_{t t}=c^{2} \Delta u+\sum_{i=1}^{n+1} b_{i}(x, t) u_{x_{i}}+c(x, t) u+F(x, t) \text { in } D_{T}^{n+1}, \\
\left.u\right|_{t=0}=g(x),\left.u_{t}\right|_{t=0}=f(x)
\end{gathered}
$$

and

$$
g(x)=f(x)=F(x, t)=0 \text { for } x \in\left\{x_{i}<0\right\} \cup\left\{x_{i}>R\right\}, \forall i=1, \ldots, n .
$$

where the term with $u_{x_{n+1}} \triangleq u_{t}$ is describing the attenuation and $c=$ const. $>0$. In acoustic, $c$ is the speed of sound. We assume that all coefficients of the equation (1.1) are bounded in $R^{n}$. Also, let

$$
g \in H^{2}\left(R^{n}\right), f \in H^{1}\left(R^{n}\right), F \in L_{2}\left(D_{T}^{n+1}\right) .
$$

Hence, for any pair of initial conditions satisfying (2.3), (2.4) there exists unique solution $u \in H^{2}\left(D_{T}^{n+1}\right)$ of the problem (2.1), (2.2) [15]. We also consider a more general case of a hyperbolic inequality. In other words, we replace the equation (2.1) with

$$
\left|u_{t t}-c^{2} \Delta u\right| \leq M\left[\left|\nabla_{x, t} u\right|+|u|+|F(x, t)|\right] \text { in } D_{T}^{n+1}
$$

Denote $P_{i}=\left\{x_{i}=0\right\} \cap \bar{O}_{n}$ the 'positive' part of the coordinate plane $\left\{x_{i}=0\right\}$. Let $\Omega(T, R) \subset O_{n}$ be a certain cube whose $n$ sides are parts of surfaces $P_{i}$, i.e., $\partial \Omega(T, R) \cap P_{i}=$ $P_{i}^{\prime} \neq \varnothing, \forall i=1, \ldots, n$. We will specify this cube later. Denote

$$
\begin{aligned}
& Q_{T}=\Omega(R, T) \times(0, T), \\
& \Gamma_{T}=\left(\bigcup_{i=1}^{n} P_{i}^{\prime}\right) \times(0, T), \\
& S_{T}=\partial \Omega(T, R) \times(0, T) .
\end{aligned}
$$

We consider the following

Stability Problem. Let the function $u \in H^{2}\left(D_{T}^{n+1}\right)$ satisfies conditions (2.2), (2.5). Suppose that either $g(x) \equiv 0$ and the function $f(x)$ is unknown or $f(x) \equiv 0$ and the function $g(x)$ is unknown. Also, assume that conditions (2.3) and (2.4) hold. Derive the Lipschitz stability estimate for the function $u(x, t)$ in the cylinder $Q_{T}$ given the lateral Cauchy data $\varphi$ and $\psi$,

$$
\left.u\right|_{\Gamma_{T}}=\varphi(x, t),\left.\frac{\partial u}{\partial \nu}\right|_{\Gamma_{T}}=\psi(x, t),
$$

where $\nu$ is the outward normal vector at $\Gamma_{T}$.

We also consider the following

Inverse Problem. Suppose that the function $u \in H^{2}\left(D_{T}^{n+1}\right)$ satisfies conditions (2.1), (2.2) and either $g(x) \equiv 0$ and the function $f(x)$ is unknown or $f(x) \equiv 0$ and the function $g(x)$ is unknown. Determine the unknown initial condition $f(x)$ or $g(x)$ given the lateral Cauchy data (2.6). 
We will demonstrate that the solution of the Stability Problem provides the Lipschitz stability estimate for the Inverse Problem, which, in particular implies the uniqueness of the latter. It was shown in [8], [13] and [14] that in the problem of computational time reversal one is supposed to reconstruct the time reversed wave field $v(x, \tau) \triangleq u(x, T-\tau)$ for $(x, \tau) \in Q_{T}(n=3)$, given the lateral Cauchy data. Hence, if a numerical method reconstructs the function $u(x, t)$ from conditions (2.1), (2.6), then it also solves both the problem of the computational time reversal and the Inverse Problem. As soon as the initial conditions (1.2) are reconstructed, the problem of time reversal can be solved via the solution of the standard Cauchy problem (1.1), (1.2).

\section{$3 \quad$ Lipschitz Stability}

Because of (2.3), consider the cube $K(R)$ in the octant $O_{n}$,

$$
K(R)=\left\{0<x_{i}<R, i=1, \ldots, n\right\} \subset O_{n} .
$$

First, we establish the following

Lemma. Let the function $u \in H^{2}\left(D_{T}^{n+1}\right)$ satisfies conditions (2.2), (2.5), and conditions (2.2)-(2.4) hold. Denote

$$
G(R, T)=\left\{x: x \in O_{n}, \operatorname{dist}(x, K(R))>c T\right\} \times(0, T),
$$

where dist $(x, K(R))$ denotes the Hausdorf distance. Then $u(x, t)=0$ for all $(x, t) \in$ $G(R, T)$.

Proof. Let $x_{0} \in\left\{x: x \in O_{n}\right.$, dist $\left.(x, K(R))>c T\right\}$ be an arbitrary point. Consider the cone

$$
\Phi\left(x_{0}, T\right)=\left\{(x, t) \in D_{T}^{n+1}:\left|x-x_{0}\right|-c(T-t)<0\right\} .
$$

Then denoting $p(x, t):=u_{t t}-c^{2} \Delta u$ and applying the standard method of energy estimates [15], we obtain that $u(x, t)=0$ in $\Phi\left(x_{0}, T\right)$. Hence, $u\left(x_{0}, t\right)=0$ for $t \in(0, T)$.

Let $\alpha$ be a small positive number which will be specified below. Denote

$$
\Omega(R, T):=K(R+\alpha+c T)=\left\{0<x_{i}<R+\alpha+c T, i=1, \ldots, n\right\} \subset O_{n},
$$

Then the boundary $\partial \Omega(R, T)$ of this cube consists of two parts, $\partial \Omega(R, T)=B_{1} \cup B_{2}$, where

$$
\begin{gathered}
B_{1}=\bigcup_{i=1}^{n} P_{i}^{\prime}=\bigcup_{i=1}^{n}\left\{x_{i}=0,0<x_{j}<R+\alpha+c T, \forall j \neq i\right\}, \\
B_{2}=\bigcup_{i=1}^{n}\left\{x_{i}=R+\alpha+c T, 0<x_{j}<R+\alpha+c T, \forall j \neq i\right\} .
\end{gathered}
$$

Hence,

$$
\Gamma_{T}=B_{1 T}:=B_{1} \times(0, T) \text { and } S_{T}=B_{1 T} \cup B_{2 T}, \text { where } B_{2 T}=B_{2} \times(0, T) .
$$


By Lemma,

$$
\left.u\right|_{B_{2 T}}=\left.\frac{\partial u}{\partial \nu}\right|_{B_{2 T}}=0 .
$$

And by (2.6)

$$
\left.u\right|_{B_{1 T}}=\varphi(x, t),\left.\frac{\partial u}{\partial \nu}\right|_{B_{1 T}}=\psi(x, t)
$$

Let

$$
T>\frac{\sqrt{n}(R+\alpha)}{(2-\sqrt{n}) c} .
$$

Note that $2-\sqrt{n}>0$ since $n=2,3$. Since

$$
\operatorname{diam}[\Omega(R, T)]=\sqrt{n}(R+\alpha+c T),
$$

then by (3.3)

$$
\operatorname{diam}[\Omega(R, T)]<2 c T .
$$

Hence, we can now apply the Lipschitz stability estimate for a hyperbolic equation/inequality with the lateral Cauchy data in a finite domain.

Theorem 1. Let the function $u \in H^{2}\left(D_{T}^{n+1}\right)$ satisfies conditions (2.2), (2.5), and conditions (2.2)-(2.4) hold. Suppose that $n=2,3$ and the inequality (3.5) is true for an $\alpha>0$. Then the following Lipshcitz stability estimate is valid

$$
\|u\|_{H^{1}\left(Q_{T}\right)} \leq C\left[\|\varphi\|_{H^{1}\left(\Gamma_{T}\right)}+\|\psi\|_{L_{2}\left(\Gamma_{T}\right)}+\|F\|_{L_{2}\left(Q_{T}\right)}\right]
$$

where the positive constant $C=C\left(T, R, \alpha, Q_{T}, M, c\right)$.

Proof. Let $Q_{T}^{-}=\left\{(x, t):(x,-t) \in Q_{T}\right\}$. In the case $g(x)=0$, consider the odd extension of the function $u(x, t)$ in the domain $Q_{T}^{-}$. If, however $f(x)=0$, then consider the even extension in this domain. In both cases the resulting function $\widetilde{u}(x, t) \in H^{2}\left(Q_{T} \cup Q_{T}^{-}\right)$. The rest of the proof follows immediately from (3.1), (3.2) and the Lipschitz stability estimate for the finite domain.

Consider now the Inverse Problem.

Theorem 2. Let the function $u \in H^{2}\left(D_{T}^{n+1}\right)$ satisfies conditions (2.2), (2.5), and conditions (2.2)-(2.4) hold. Suppose that $n=2,3$ and the inequality (3.4) is true for an $\alpha>0$. Consider two cases of the above Inverse Problem. In the first case the function $g(x) \equiv 0$ and the function $f(x)$ is unknown. In the second case the function $g(x)$ is unknown and the function $f(x) \equiv 0$. Then the following Lipschitz stability estimates are valid: (1) in the case one

$$
\|f\|_{L_{2}(\Omega(R, T))} \leq C_{1}\left[\|\varphi\|_{H^{1}\left(\Gamma_{T}\right)}+\|\psi\|_{L_{2}\left(\Gamma_{T}\right)}+\|F\|_{L_{2}\left(Q_{T}\right)}\right]
$$

and (2) in the second case

$$
\|g\|_{L_{2}(\Omega(R, T))} \leq C_{1}\left[\|\varphi\|_{H^{1}\left(\Gamma_{T}\right)}+\|\psi\|_{L_{2}\left(\Gamma_{T}\right)}+\|F\|_{L_{2}\left(Q_{T}\right)}\right]
$$

where the positive constant $C_{1}$ has the same meaning as the constant $C$ in Theorem 1. 
Proof. The second case follows immediately from Theorem 1. Hence, we consider only the first case. By Theorem 1 and the mean value theorem for integrals, there exists a number $\tau \in(0, T)$ such that

$$
\left[\int_{\Omega(R, T)}\left[u^{2}+|\nabla u|^{2}+u_{t}^{2}\right](x, \tau) d x\right]^{1 / 2} \leq \frac{C}{T}\left[\|\varphi\|_{H^{1}\left(\Gamma_{T}\right)}+\|\psi\|_{L_{2}\left(\Gamma_{T}\right)}+\|F\|_{L_{2}\left(Q_{T}\right)}\right] .
$$

Since a hyperbolic equation can be solved both upwards and downwards with respect to time, then the rest of the proof follows from the standard energy estimate.

\section{The Quasi-Reversibility Method}

We now present a stable numerical method for the reconstruction of the function $u(x, t)$ satisfying the equation (2.1) in the domain $Q_{T}=\Omega(R, T) \times(0, T)$ from conditions (2.3), (2.6), (3.1) and (3.2) assuming that either $f(x) \equiv 0$ or $g(x) \equiv 0$ and these initial conditions are unknown. It was shown in [] that a modification of the QRM should work for this problem, since the lateral Cauchy data are given on the entire boundary of the cube $\Omega(R, T)$. Thus, we briefly outline the QRM here for the convenience of the reader.

Denote

$$
L u:=u_{t t}-c^{2} \Delta u-\sum_{i=1}^{n+1} b_{i}(x, t) u_{x_{i}}-c(x, t) u .
$$

Hence, we have to find a function $u \in H^{2}\left(Q_{T}\right)$ satisfying boundary conditions (3.1), (3.2) and the equation

$$
L u=F(x, t) \text { in } Q_{T} .
$$

Let $\varepsilon$ be a positive regularizing parameter. Consider the Tikhonov functional (see, e.g., [20] for this functional)

$$
J_{\varepsilon}(v)=\|L v-F\|_{L_{2}\left(Q_{T}\right)}^{2}+\varepsilon\|v\|_{L_{2}\left(Q_{T}\right)}^{2} .
$$

We minimize the functional $J_{\varepsilon}$ over all functions $v \in H^{2}\left(Q_{T}\right)$ satisfying boundary conditions (3.1), (3.2). Its minimizer $u_{\varepsilon}$ satisfies the following conditions

$$
\begin{gathered}
\left(L u_{\varepsilon}, L w\right)+\varepsilon(u, w)=(F, L w), \forall w \in H_{0}^{2}\left(Q_{T}\right), \\
\left.u_{\varepsilon}\right|_{S_{T}}=\xi(x, t),\left.\frac{\partial u_{\varepsilon}}{\partial \nu}\right|_{S_{T}}=\eta(x, t),
\end{gathered}
$$

where $($,$) denotes the scalar product in L_{2}\left(Q_{T}\right), S_{T}=\partial \Omega(R, T) \times(0, T)$ and

$$
\begin{gathered}
H_{0}^{2}\left(Q_{T}\right)=\left\{w \in H^{2}\left(Q_{T}\right):\left.w\right|_{S_{T}}=\left.\frac{\partial w}{\partial \nu}\right|_{S_{T}}=0\right\}, \\
(\xi(x, t), \eta(x, t))=\left\{\begin{array}{l}
(\varphi(x, t), \psi(x, t)) \text { for }(x, t) \in \Gamma_{T}, \\
0 \text { for }(x, t) \in B_{2 T}
\end{array}\right.
\end{gathered}
$$


The boundary value problem (4.4), (4.5) is the weak formulation of the QRM. This problem can be solved by the Finite Element Method. The solution of this problem represents an approximate solution of both the above inverse problem and the problem of time reversal. The latter is because the time reversed wave field is $v(x, \tau)=u(x, T-\tau)$ for $(x, \tau) \in Q_{T}$.

To formulate the uniqueness, existence and convergence result for the QRM, we need to assume that both the data and the right hand side of the equation (4.2) are given with a certain error $\sigma$, i.e.,

$$
\left\|\varphi_{\sigma}-\varphi\right\|_{H^{1}\left(\Gamma_{T}\right)}+\left\|\psi_{\sigma}-\psi\right\|_{L_{2}\left(\Gamma_{T}\right)}+\left\|F_{\sigma}-F\right\|_{L_{2}\left(Q_{T}\right)} \leq \sigma,
$$

where $\sigma$ is a small positive parameter, functions $\varphi, \psi$ and $F$ correspond respectively to the exact data and the exact right hand side (which are never known in practice) and functions $\varphi_{\sigma}, \psi_{\sigma}$ and $F_{\sigma}$ are those given with an error. This is actually the Tikhonov concept of ill-posed problems, see, e.g., [20] for more details about this concept. Hence, we now have

$$
(\xi(x, t), \eta(x, t)):=\left(\xi_{\sigma}(x, t), \eta_{\sigma}(x, t)\right)=\left\{\begin{array}{l}
\left(\varphi_{\sigma}(x, t), \psi_{\sigma}(x, t)\right) \text { for }(x, t) \in \Gamma_{T}, . \\
0 \text { for }(x, t) \in B_{2 T}
\end{array} .\right.
$$

The following theorem is a reformulation of Theorem 2.5.4 of [14]

Theorem 3. Suppose, there exists a function $\Psi_{\sigma}(x, t) \in H^{2}\left(Q_{T}\right)$ such that

$$
\left.\Psi_{\sigma}\right|_{S_{T}}=\xi_{\sigma}(x, t), \frac{\partial \Psi_{\sigma}}{\partial \nu S_{T}}=\eta_{\sigma}(x, t) .
$$

Then for any $\varepsilon>0$ there exists a unique solution $u_{\varepsilon} \in H^{2}\left(Q_{T}\right)$ of the equation (4.4) with the boundary data (4.8) and

$$
\left\|u_{\varepsilon}\right\|_{H^{2}\left(Q_{T}\right)} \leq \frac{A}{\sqrt{\varepsilon}}\left[\left\|\Psi_{\sigma}\right\|_{H^{2}\left(Q_{T}\right)}+\left\|F_{\sigma}\right\|_{L_{2}\left(Q_{T}\right)}\right],
$$

where the positive constant $A$ depends only on coefficients of the operator $L$ and the domain $Q_{T}$ but it is independent on parameters $\varepsilon$ and $\sigma$. Assume, in addition that conditions (3.4), (3.5) and (4.7) are fulfilled, there exists an exact solution $u \in H^{2}\left(Q_{T}\right)$ of the equation (4.2) with the boundary data (3.1), (3.2), and the boundary data (4.5) have the form (4.8). Then the following estimate holds

$$
\left\|u-u_{\varepsilon}\right\|_{H^{1}\left(Q_{T}\right)} \leq A\left(\sigma+\sqrt{\varepsilon}\|u\|_{H^{2}\left(Q_{T}\right)}\right) .
$$

Hence, taking the regularizing parameter $\varepsilon:=\varepsilon(\sigma)=a \sigma^{2}$, where the positive constant a is independent on $\sigma$, one obtains convergence of the approximate solution $u_{\varepsilon(\sigma)}$ to the exact one when the error $\sigma$ in the data approaches zero,

$$
\lim _{\sigma \rightarrow 0^{+}}\left\|u-u_{\varepsilon(\sigma)}\right\|_{H^{1}\left(Q_{T}\right)}=0 .
$$




\section{$5 \quad$ Refocusing}

First, we introduce a formal definition of refocusing. Let $v(x, \tau)=u(x, T-\tau)$ be the time reversed wave field originated by point-like sources at $\{t=0\}$. Intuitively refocusing means that the function $v(x, \tau)$ at $\{\tau=T\}$ should replicate the behavior of the original wave field $u(x, t)$ at $\{t=0\}$. Our definition of refocusing reflects this thought. In this section $R^{n}:=R^{3}$.

Suppose that in $(2.2) g(x) \equiv 0$ and the function $f(x)$ is a linear combination of smooth $\delta$-like functions, which models the superposition of $\delta$-like pulses, i.e.,

$$
f(x)=\sum_{k=1}^{N} B_{i} h_{\varepsilon}\left(x-x^{i}\right),\left\{x^{i}\right\}_{i=1}^{N} \subset O_{3},\left|x^{i}\right|+\varepsilon<R
$$

where $x_{i}$ are the points where sources are, $B_{i}$ are certain numbers, $\varepsilon$ is a small positive number and the function $h_{\varepsilon}(x)$ is such that

$$
\begin{gathered}
h_{\varepsilon}(x) \geq 0 \text { in } R^{3}, h_{\varepsilon}(x)=0 \text { for }|x|>\varepsilon, h_{\varepsilon}(x) \in C^{\infty}\left(R^{n}\right), \\
\int_{|x|<\varepsilon} h_{\varepsilon}(x) d x=1 .
\end{gathered}
$$

Examples of such functions are well known. The Cauchy-Schwarz inequality and (5.2) imply that

$$
\left\|h_{\varepsilon}\right\|_{L_{2}(|x|<\varepsilon)} \geq \sqrt{\frac{3}{4 \pi}} \varepsilon^{-3 / 2} .
$$

Naturally, we assume that sources are sufficiently well separated, so that

$$
\left|x_{i}-x_{j}\right|>\varepsilon \text { for } i \neq j \text {. }
$$

We now assume that the function $u(x, t) \in H^{2}\left(D_{T}^{4}\right)$ is the solution of the equation(2.1) with the initial conditions

$$
\left.u\right|_{t=0}=0,\left.u_{t}\right|_{t=0}=f(x) .
$$

Let

$$
\sigma \in\left(0, \sqrt{\frac{3}{4 \pi}} \varepsilon^{-3 / 2}\right)
$$

be a small positive number characterizing the level of the error in the lateral data (3.2). Let the function $u$ satisfies the exact boundary data (3.2) and the exact right hand side $F$. As a rule, in a time reversed experiment the lateral Cauchy and the right hand side are given with an error and the exact data are unknown. Hence, similarly with the previous section we assume (4.7) and (4.8).

Definition. Let the function $f(x)$ has the form (5.1) and the function $u \in H^{2}\left(D_{T}^{4}\right)$ be the solution of the standard Cauchy problem (2.1), (5.5). Let $u_{\sigma} \in H^{2}\left(Q_{T}\right)$ be a solution of the equation (2.1) with $F:=F_{\sigma}$ and boundary conditions

$$
\left.u_{\sigma}\right|_{\Gamma_{T}}=\varphi_{\sigma}(x, t),\left.\quad \frac{\partial u_{\sigma}}{\partial \nu}\right|_{\Gamma_{T}}=\psi_{\sigma}(x, t),
$$




$$
\left.u_{\sigma}\right|_{B_{2 T}}=\left.\frac{\partial u_{\sigma}}{\partial \nu}\right|_{B_{2 T}}=0
$$

and (4.7) holds. We say that the time reversed wave field $v(x, \tau)=u_{\sigma}(x, T-\tau)$ refocuses at $\tau=T$ near the source position $x^{i}$ in the ball $\left\{x:\left|x-x^{i}\right|<\varepsilon\right\}$ and with the accuracy $\sigma$ if the following inequalities hold

$$
\begin{gathered}
\left\|u_{\sigma}(x, 0)\right\|_{L_{2}\left(\left|x-x^{i}\right|<\varepsilon\right)} \leq C_{1} \sigma \\
\left\|\partial_{t} u_{\sigma}(x, 0)\right\|_{L_{2}\left(\left|x-x^{i}\right|<\varepsilon\right)} \geq\left|B_{i}\right| \sqrt{\frac{3}{4 \pi}} \varepsilon^{-3 / 2}-C_{1} \sigma,
\end{gathered}
$$

where $C_{1}$ is positive constants independent on parameters $\varepsilon, \sigma$ and numbers $B_{i}$.

Theorem 4. Let the function $f(x)$ has the form (5.1) and the function $u \in H^{2}\left(D_{T}^{4}\right)$ be the solution of the standard Cauchy problem (2.1), (5.5). Suppose that conditions (3.4), (3.5) hold. Let $u_{\sigma} \in H^{2}\left(Q_{T}\right)$ be a solution of the equation (2.1) with $F:=F_{\sigma}$ and boundary conditions (5.6), (5.7). Then there exists a positive number $C_{1}$ depending only on coefficients of the equation (2.1) and the domain $Q_{T}$ such that estimates (5.8), (5.9) hold for all $\sigma \in$ $\left(0, \sigma_{0}\right)$, where

$$
\sigma_{0} \in\left(0, \sqrt{\frac{3}{4 \pi}} \varepsilon^{-3 / 2}\right)
$$

is a sufficiently small positive number. In other words, refocusing takes place.

Proof. By Theorem 2 and (4.7)

$$
\begin{gathered}
\left\|u(x, 0)-u_{\sigma}(x, 0)\right\|_{L_{2}(\Omega(R, T))} \leq C_{1} \sigma, \\
\left\|\partial_{t} u(x, 0)-\partial_{t} u_{\sigma}(x, 0)\right\|_{L_{2}(\Omega(R, T))} \leq C_{1} \sigma .
\end{gathered}
$$

Since $u(x, 0)=0$, then (5.10) implies (5.8). Now, by (5.11)

$$
\left\|\partial_{t} u_{\sigma}(x, 0)\right\|_{L_{2}\left(\left|x-x^{i}\right|<\varepsilon\right)} \geq\left\|\partial_{t} u(x, 0)\right\|_{L_{2}\left(\left|x-x^{i}\right|<\varepsilon\right)}-C_{1} \sigma .
$$

Hence, (5.1)-(5.4) imply that

$$
\left\|\partial_{t} u_{\sigma}(x, 0)\right\|_{L_{2}\left(\left|x-x^{i}\right|<\varepsilon\right)} \geq\left|B_{i}\right| \sqrt{\frac{3}{4 \pi}} \varepsilon^{-3 / 2}-C_{1} \sigma .
$$

\section{Acknowledgment}

The author is grateful to Professor A. Yagola for encouraging him to work on this paper. This work was partially supported by the NATO grant PDD(CP)-(PST.NR.CLG 980631) and by a research grant from the University of North Carolina at Charlotte.

\section{References}


1. G. Bal and L. Ryzhik, Time reversal and refocusing in random media, SIAM J. Appl. Math., 63, 1475-1498, 2003.

2. C. Bardos and M. Fink, Mathematical foundations of the time reversal mirror, Asymptotic Analysis, 29, 157-182, 2002.

3. C. Bardos, A mathematical and deterministic analysis of the time-reversal mirror, Inside Out: Inverse Problems, MSRI Publications, 47, 377-395, 2003.

4. P. Blomgren, G.C. Papanicolaou and H. Zhao, Super-resolution in time-reversal acoustics, J. Acoustical Soc. of America, 111, 5139-5164, 2003.

5. L.Borcea, G.C. Papanicolaou, C. Tsogka and J. Berryman, Imaging and time reversal in random media, Inverse Problems, 18, 1247-1279, 2002.

6. M. Fink and C. Prada, Acoustic time-reversal mirrors, Inverse Problems, 17, R1-R38, 2001.

7. J.-P. Fouque and K. Solna, Time-reversal aperture enhancement, Multiscale Modeling and Sumul., 1, 239-259, 2003.

8. B.L.G. Jonsson, M. Gustafsson, Y. B. Weston and M.V. De Hoop, Retrofocusing of acoustic wave fields by iterated time reversal, SIAM J. Appl. Math., 64, 1954-1986, 2004.

9. V. Isakov and M. Yamamoto, Carleman estimate with the Neumann boundary conditions and its applications to the observability inequality in inverse problems, Contemporary Math., 268, 601-618, 2000.

10. M. Kazemi and M.V. Klibanov, Stability estimates fro ill-posed Cauchy problems involving hyperbolic equations and inequalities, Applicable Anal., 50, 93-102, 1993.

11. M.V. Klibanov and J. Malinsky, Newton-Kantorovich method for 3-dimensional potential inverse scattering problem and stability of the hyperbolic Cauchy problem with time dependent data, Inverse Problems, 7, 577-595, 1991.

12. M.V. Klibanov and Rakesh, Numerical solution of a timelike Cauchy problem for the wave equation, Math. Meth. in Appl. Sci., 15, 559-570, 1992.

13. M.V. Klibanov and A. Timonov, On the mathematical treatment of time reversal, Inverse Problems, 19, 1280-1299, 2003.

14. M.V. Klibanov and A. Timonov, Carleman Estimates for Coefficient Inverse Problems and Numerical Applications, VSP, Utrecht, 2004.

15. O.A. Ladyzhenskaya, Boundary Value Problems of Mathematical Physics, Springer, Berlin, 1985.

16. I. Lasiecka, R. Triggiani and X. Zhang, Nonconservative wave equations with unobserved Neumann B.C.: Global uniqueness and observability in one shot, Contemporary Math., 268, 227-325, 2000.

17. I. Lasiecka, R. Triggiani and X. Zhang, Global uniqueness, observability and stabilization of nonconservative Schrödinger equations via pointwise Carleman estimates. Part I: $H^{1}(O)$-estimates, J. Inv. and Ill-Posed Problems, 12, 43-123, 2004

18. I. Lasiecka, R. Triggiani and X. Zhang, Global uniqueness, observability and stabilization of nonconservative Schrödinger equations via pointwise Carleman estimates. Part II: $L_{2}(O)$-estimates, J. Inv. and Ill-Posed Problems, 12, 183-231, 2004. 
19. R.Lattes and J.-L. Lions, The Method of Quaisreversibility: Applications to Partial Differential Equations, Elsevier, New York, 1969.

20. A.N. Tikhonov, A.S. Leonov and A.G. Yagola, Nonlinear Ill-Posed Problems, Volumes 1 and 2, Chapman and Hall, London, 1998. 